\title{
ANÁLISE WAVELET APLICADA NA MUDANÇA DE ESCALA EM PERFIS GEOFÍSICOS DO CAMPO DE NAMORADO
}

\author{
ADALBERTO DA SILVA ${ }^{1}$, JOÃO MARCELO B. PROTÁZIO² \& ARMANDO Z. REMACRE ${ }^{2}$
}

\begin{abstract}
WAVELET ANALYSIS APPLIED TO SCALE CHANGES IN GEOPHYSICAL PROFILES OF THE NAMORADO FIELD Scale transference is a fundamental technique in the oil industry. The development of new methods that do integrate high vertical resolution / low areal or volumetric distribution well data with low vertical resolution / high areal or volumetric distribution seismic data are a real challenge for the industry.

In this article we present an application of the Wavelet Transform as an upscaling method. Density and sonic logs from 7 wells at the Namorado Field (Campos Basin, RJ, Brazil) were filtered down to a seismic-compatible scale using cutting frequencies derived from spectral analysis and variogram analysis as a control tool to the filtering process.

The results we present on this article clearly show the potential of this integrating technique as a good basis for scale transference.
\end{abstract}

Keywords: wavelet analysis, variogram, upscaling, well log

\begin{abstract}
Resumo A mudança de escala é um procedimento fundamental na prospecção e desenvolvimento de jazidas de petróleo. As estratégias para integração de dados de poço (caracteristicamente, com grande resolução vertical e pequena distribuição em área ou volume) com informações sísmicas (de grande distribuição em área ou volume mas baixa resolução vertical) constituem um grande desafio para a indústria. Neste trabalho, apresentamos uma aplicação da Transformada Wavelet na filtragem de informações de perfis geofísicos de poço (densidade e sônico), de modo a transferir as informações geológicas do reservatório para escalas compatíveis com a aquisição sísmica. Foram processados 7 poços do Campo de Namorado (Bacia de Campos, RJ), com freqüência de corte derivada de análise espectral e controle de filtragem utilizando técnicas de análise variográfica. Os resultados aqui obtidos através da aplicação de análise wavelet e variográfica, dentro de um arcabouço interpretativo geológico, demonstram de modo claro o potencial desta técnica como uma boa base para estratégias de mudança de escala.
\end{abstract}

Palavras-chave: análise wavelet, variograma, transferência de escala, filtragem de perfis de poço

INTRODUÇÃO A prospecção e desenvolvimento das jazidas de petróleo exigem a caracterização detalhada das rochas reservatórios. A geometria do corpo rochoso e a distribuição espacial das variações de propriedades como porosidade, permeabilidade, espessura, saturação em óleo, dentre outras, devem necessariamente ser conhecidas tanto para orientar os estudos exploratórios quanto para auxiliar na definição da estratégia de desenvolvimento e explotação e no gerenciamento da produção.

Várias estratégias de integração são expostas na literatura, principalmente através do uso de técnicas geoestatísticas e de inversão sismoestratigráfica, com forte condicionamento imposto pelo dado sísmico e com tratamento convencional do dado geofísico de poço (e.g., Mundim 1999).

Nos tratamentos usuais, o sinal de perfil sônico e de densidade sofrem uma conversão de escala do domínio do espaço para o domínio do tempo, são filtrados (com bandas de freqüência de corte derivadas do espectro do sinal sísmico), e convoluídos com o pulso sísmico num traço sísmico sintético. Caracteristicamente, o controle da filtragem se utiliza do sinal sísmico, com espectro mais empobrecido e de menor resolução vertical mas que, em contrapartida, apresenta a cobertura espacial mais adequada ao reconhecimento e mapeamento tridimensional das informações geológicas.

Este trabalho propõe um enfoque alternativo, decompondo e analisando o sinal de perfis de poço (utilizando a transformada wavelet), e reduzindo por filtragem a informação geológica às escalas compatíveis com a aquisição sísmica. Neste procedimento, a escala natural da formação é preservada, permitindo o controle eficaz das rotinas de filtragem com base no conhecimento da geologia do reservatório, em grande parte derivada da própria interpretação de perfis.

CONTEXTOGEOLÓGICODOCAMPODENAMORADO O Reservatório Namorado (Fig. 1) é um dos campos petrolíferos mais importantes da Bacia de Campos na área da plataforma continental. O poço pioneiro do reservatório foi perfurado em 1975 numa lâmina d'água de 166 m e para o seu desenvolvimento foram realizadas perfurações desde $110 \mathrm{~m}$ até $250 \mathrm{~m}$, já no topo do talude continental (Souza Jr. 1997). O Arenito Namorado, denominação informal desta unidade siliciclástica, caracteriza-se pelo desenvolvimento de corpos arcosianos espessos, com extensiva cimentação carbonática, e boas porosidades efetivas. O pacote sedimentar apresenta velocidades sísmicas elevadas, tanto pela cimentação dos arenitos quanto pela intercalação com corpos de marga e calcilutitos.

O Arenito Namorado ocupa a porção superior da Formação Macaé, de idade que varia entre o Albiano Superior e o Cenomaniano Inferior (Souza Jr. 1997), e é composto por brechas,

1 - ANP/PRH-11, Depto. de Geologia/LAGEMAR - UFF, Av. Litorânea, s/n, Instituto de Geociências, 4 andar, Gragoatá - UFF, Niterói, RJ, Brasil, CEP 24210-340, adalberto@igeo.uff.br

2 - Instituto de Geociências, Universidade Estadual de Campinas, UNICAMP, Caixa Postal: 6152 - CEP: 13083-970 - Campinas - SP, Brasil 
conglomerados e arenitos, intercalados a espessos pacotes de margas e camadas mais finas de argilitos e calcilutitos.

ATRANSFORMADAWAVELET E SUASAPLICAÇÕES O conceito de Transformada Wavelet (TW) foi formalizado pela primeira vez na década de 1980 em uma série de artigos de Grossman e Morlet (Hubbard 1998). Entretanto, já em 1910, o físico Alfred Haar introduzira um sistema completo de funções ortogonais com muitas das propriedades e características que fazem das wavelets uma ferramenta matemática com vasto campo de aplicações nas mais diversas ciências.

Contudo, somente a partir da segunda metade da década de 1980 , foram definidos função com rigor os conceitos que permitem compreender de forma clara a natureza deste tipo de, estabelecendo-se as suas propriedades e permitindo a construção e a geração de outras famílias de wavelets (e.g.: Meyer,1989, Mallat 1989, Daubechies 1992).

Grande parte da teoria wavelet foi desenvolvida de forma independente em vários campos do conhecimento como Matemática, Física Quântica, Engenharia Elétrica e Sísmica. Contribuições entre estes campos específicos durante os últimos dez anos têm levado a um número maior de aplicações, nas áreas de processamento e compressão de imagens, turbulência, visão humana e radar, entre outras.

Wavelets são funções-ondas com duração finita ou comprimento finito com valor médio zero.

A transformada wavelet contínua (CWT) é uma transformação integral definida como:

$$
\begin{gathered}
\psi(\text { escala, posição })=\int f(t) \phi(\text { escala }, \text { posição, } t) d t \\
\text { (equação 1) }
\end{gathered}
$$

onde $\phi$ é denominaaa ae runçao base ou runção wavelet-mãe e $\mathrm{f}(\mathrm{t})$ é o sinal que está sendo analisado.

A função $\phi$ pode ser reescrita como $\phi_{a b}(t)=\phi($ escala, posição, $t)$ ou ainda:

$$
\phi_{a b}(t)=\phi\left(\frac{t-b}{a}\right) \quad(\text { equação } 2)
$$

Pode-se observar que a transformada wavelet de um sinal a 1D produz como resultado uma matriz de coeficientes wavelet

$$
\psi(a, b)
$$

Esta distribuição no espaço $(a, b)=($ escala, posição) fornece informação sobre a evolução de componentes de diferentes escalas no tempo (ou profundidade) e é também chamada de espectro de coeficientes wavelet. $\mathrm{Na}$ figura 2 apresenta-se dois exemplos de wavelets com suas respectivas funções-escalas.
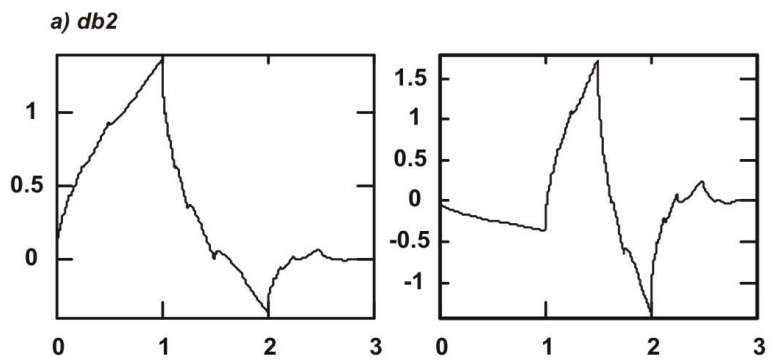

b) $d b 8$
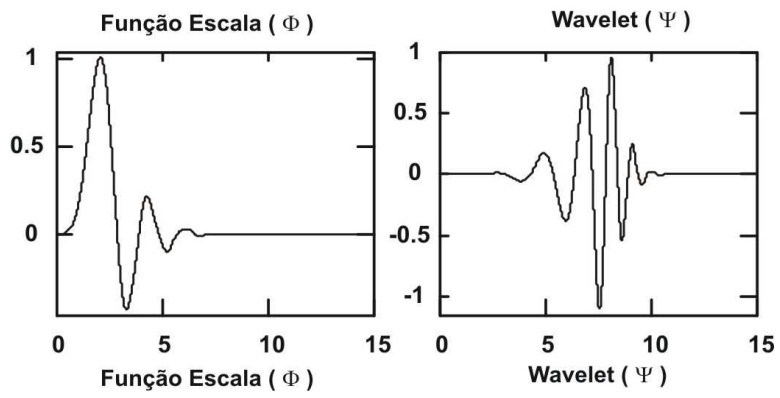

Figura 2 - Exemplos de wavelets de Daubechies e respectivas funções escalas.
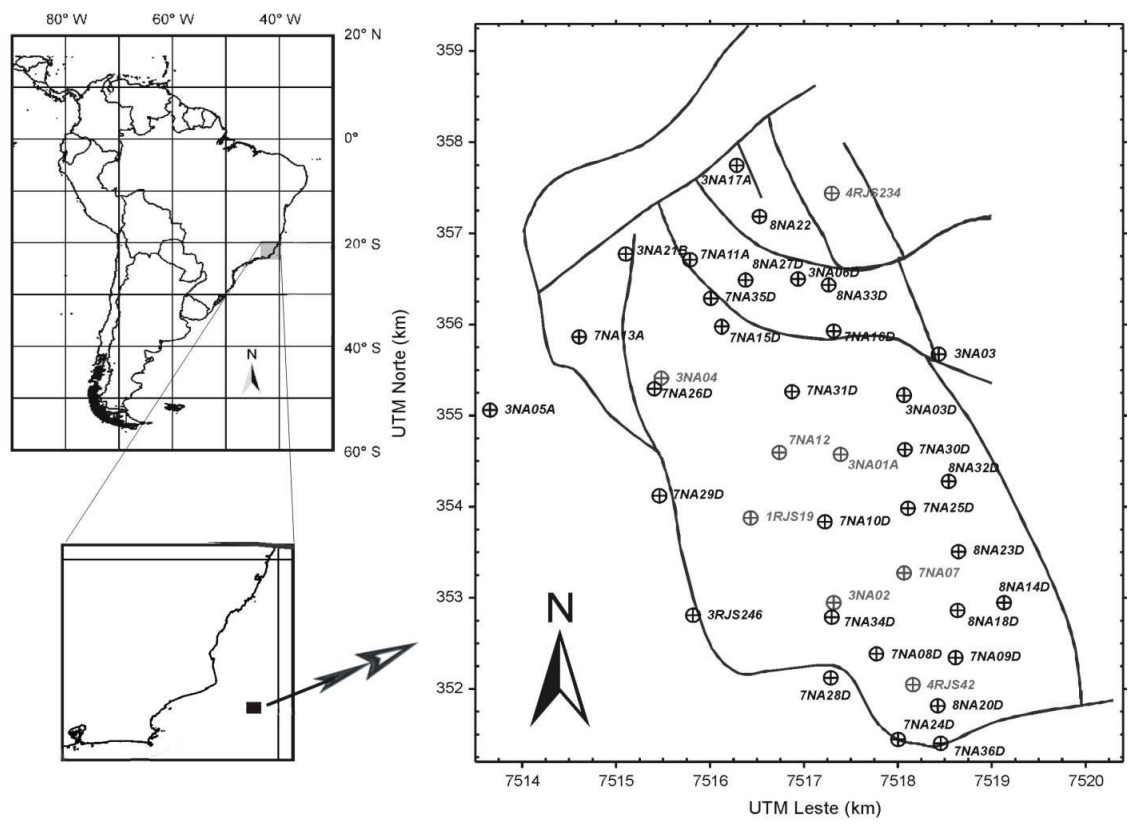

Figura 1 - Localização dos poços utilizados no Campo de Namorado. As linhas sólidas indicam os principais traços de falhas que limitam o campo. 
Como observam Torrence \& Compo (1998), a escolha da função base (ou núcleo) determina o tipo de informação que pode ser extraída de um processo. A grande limitação das metodologias baseadas na Transformada de Fourier (TF) reside justamente na representação de fenômenos não-estacionários através de sinusóides oscilam infinitamente.

Em contraposição, as wavelets são funções que oscilam num intervalo limitado (Fig. 2) e, portanto, permitem obter localização tanto no tempo quanto na freqüência. A idéia desta representação é a separação do sinal de interesse em várias partes e a análise de cada uma delas separadamente, ou seja, usar as grandes escalas para mostrar as características mais globais do sinal e as pequenas para os detalhes ou características locais do mesmo. De modo similar ao obtido por meio das séries de Fourier, uma representação de um processo através das wavelets se processa por uma expansão em uma série infinita de versões dilatadas (ou comprimidas) e transladadas de uma wavelet-mãe (ou básica), multiplicadas por um coeficiente apropriado.

Assim, a TW é capaz de revelar aspectos do sinal que não podem ser extraídos por outras técnicas de processamento, tais como tendências, pontos de descontinuidade, descontinuidades em derivadas superiores e auto-similaridade.

Do ponto de vista teórico, Graps (1995) aponta a potencialidade de aplicações das wavelets em várias áreas e demonstra que as wavelets são funções matemáticas que separam um sinal em suas componentes de diferentes freqüências e estuda cada uma das componentes com a resolução compatível para esta escala. Conseqüentemente, o método possui vantagens sobre a TF na análise de situações físicas onde o sinal contém descontinuidades e pulsos. Astaf'eva (1996) demonstra este potencial analisando vários modelos de séries, como harmônicos e fractais, e essas mesmas séries contaminadas com vários tipos de singularidades.

Num enfoque mais aplicado, Prokoph \& Barthelmes (1996) utilizam a TW na detecção e localização de descontinuidades, eventos e sequências cíclicas periódicas e caóticas em uma sucessão sedimentar marinha. Prokoph \& Agterberg (2000) aplicam a TW em perfis de raio gama para avaliar a distribuição espaço-temporal das rochas geradoras de óleo e estimar as taxas de acumulação em uma bacia sedimentar; os autores demonstram que o método é sensível para detecção automática e distinção de descontinuidades abruptas (tais como falhas e discordâncias), ciclicidade e mudanças graduais na taxa de sedimentação.

Liu (1996) analisa o espectro obtido através da TW de um conjunto de dados de ventos oceânicos, coletados durante o programa SWADE (Surface Wave Dynamics Experiment) de 1990, obtendo novos resultados que não seriam evidentes com a utilização da metodologia tradicional através da TF. De maneira semelhante, Torrence \& Compo (1998) apresentam resultados importantes no tratamento de uma série temporal relativa ao Efeito El Niño, inclusive analisando comparativamente os resultados da análise wavelet e de Fourier.

Metodologias de transferência de escala ou upscaling também têm-se valido da análise wavelet (e.g. Chu et al. 1996, Kumar et al.,1997) como alternativa às numerosas técnicas existentes na literatura especializada. A natureza das heterogeneidades multiescalas inerentes a reservatórios de petróleo, as propriedades de rocha e fluido presentes, que variam com as escalas de heterogeneidade, e a necessidade de gerar modelos discretos, no mais das vezes de escala decamétrica, indicam que o método de decomposição multi-resolução (ou multiescala) é adequado para realizar o upscaling de propriedades de rochas e fluxo nesses reservatórios.

Mais recentemente, Jansen \& Kelkar (1998) também mostram que modelos geológicos gerados em escala de detalhe determinam modelos de reservatório contendo centenas de milhares de blocos de discretização, inviáveis para a simulação de reservatórios. Para diminuir o esforço computacional foi utilizada a TW para realizar o upscaling dos valores das propriedades em escala mais detalhada para uma escala maior em 2 ou 3 dimensões. Estudando a utilização de dados de produção e correlação cruzada entre pares de poços na obtenção de direções de fluxo em reservatórios, os autores apontam as dificuldades para o cálculo deste parâmetro devido à natureza não linear e não estacionária das relações entre poços. A TW é mostrada, então, como uma nova ferramenta que, em contraste com a TF, permite o tratamento de dados não estacionários, abrindo novas possibilidades na obtenção de correlações cruzadas mais robustas e na utilização destes dados para a determinação mais consistente do regime de fluxo em cada poço e da sua influência em poços vizinhos.

Oliver et al.(2000), de forma pioneira, aplicam decomposição através da TW para descrever e localizar um amplo espectro de freqüências simultaneamente e, então, filtrá-las com decomposição multi-resolução, comparando os resultados com a filtragem obtida utilizando Krigagem Fatorial.

MÉTODOS UTILIZADOS Inicialmente, escolheu-se uma família de wavelets ortogonais para a decomposição multi-resolução do sinal (Daubechies 8 ou db8, Fig. 2) com características de regularidade e suavidade adequadas ao sinal de perfil.

Como a representação do sinal guarda estreita relação com a função-mãe escolhida, optou-se por uma wavelet assimétrica, com um número elevado de momentos e bastante suave de modo a preservar algumas características importantes do depósito turbidítico: intervalos marcantes em granodecrescência ascendente; pulsos deposicionais conservativos (sem erosão basal significativa) e relativamente auto-similares; superposição de diversas freqüências de laminação com algum grau de ciclicidade; e com pseudo-freqüência compatível com as espessura média decamétrica dos pacotes singulares estudados. Evidentemente, esta opção sacrifica relativamente a resolução do método para localização de transientes e limites de para-seqüências; para tal objetivo, uma wavelet como Haar (ou db1) seria mais adequada.

Adotou-se a mesma freqüência de corte de Silva \& Remacre (2000), obtida por análise espectral para o mesmo conjunto de perfis, e correspondente ao comprimento de onda $15 \mathrm{~m}$. Esta janela de resolução também é compatível com as freqüências sísmicas usuais, que correspondem a uma resolução vertical de 25 a $50 \mathrm{~m}$ para as maiores velocidades da formação (Silva \& Remacre2 000).

A etapa ou nível da decomposição escolhido foi o 5, resultando numa pseudo-freqüência de $0.1048 \mathrm{~Hz}$ para a wavelet $\mathbf{d b 8}$. Como o perfil está originalmente amostrado a cada $20 \mathrm{~cm}$, o comprimento de onda correspondente é de cerca de $10 \mathrm{~m}$, retirando a maior parte dos eventos do sinal com comprimento de onda inferior a $10 \mathrm{~m}$, preservando grande parte da escala natural da formação como indicado por Silva \& Remacre (2000).

Para controle do processo de filtragem, foi calculado o variograma para cada etapa da decomposição multi-resolução e para cada um dos sinais resultantes (aproximações e detalhes), e analisaram-se as estruturas do sinal. Adicionalmente, calculou-se a média e variância.

A metodologia foi aplicada à maioria dos poços utilizados no trabalho de Silva \& Remacre (2000), que utiliza também uma forma 
alternativa de realizar este procedimento a partir da TF. Neste trabalho empregou-se um algoritmo desenvolvido especificamente para este propósito (Protázio2 002) utilizando a linguagem de programação MATLABC, da Mathworks.

RESULTADOS OBTIDOS E ANÁLISE Utilizou-se 7 poços verticais (NA01, NA02, NA04, NA07, NA12, RJS42 e RJS234), com mudança de escala efetuada para os atributos densidade total (rhoB) e tempo de trânsito (dT), fundamentais na confecção de sismogramas sintéticos e na correlação sísmica/perfil de poço. Os sinais de perfis foram filtrados e analisados comparativamente com os sinais originais, levando-se em conta as principais características das rochas-reservatórios de acordo com seu posicionamento no pacote turbidítico.

A condição limite imposta à filtragem, resultado das análises de Silva \& Remacre (2000), foi controlada através da geração e análise dos variogramas do sinal original e dos sinais resultantes. A ausência de estruturas imbricadas no variograma do sinal resultante para distâncias menores ou iguais a $15 \mathrm{~m}$ indica que a maioria dos eventos do sinal com comprimento de onda correspondentes foi retirada por filtragem (Fig. 3).

Os principais parâmetros estatísticos, sintetizados na Tabela 1, apontam comportamentos variados dos sinais (original e filtrados) ao longo do reservatório.

Não se observou nenhuma variação no valor do parâmetro média (não listado na tabela) antes e após a aplicação dos filtros, sugerindo uma forte componente estacionária no sinal original. Este aparente paradoxo (uma vez que, já na simples visualização, os perfis de poço apresentam claras indicações de quebras e transiência) pode ser atribuído à ciclicidade do processo deposicional: a sucessão de eventos turbidíticos com assinaturas similares para cada conjunto individualizado, separados por um claro contraste das propriedades petrofísicas das frações mais lamosas no topo de uma para-seqüência e mais arenosas da base da seguinte. Eventualmente, sinais de perfis muito ruidosos também podem apresentar comportamento semelhante mas a análise de outros parâmetros estatísticos (Fig. 3, por exemplo) não confirma o elevado grau de aleatoriedade do sinal.

A redução da variância do sinal filtrado em relação ao original (Tabela 1) indica a degradação imposta pela mudança de escala.

De modo geral, para os comprimentos de onda adotados para corte, o sinal de densidade total é bastante preservado, como indica a redução percentual da variância, exceto em NA02 (Fig. 4) e RJS234. No primeiro caso, a predominância de níveis finamente laminados de arenitos e margas no reservatório e freqüentes níveis cimentados, relacionados à posição relativamente distal no corpo turbidítico deste poço (Fig. 5, Silva 2001), introduz uma forte componente de alta freqüência que foi simplesmente descartada pela estratégia de filtragem. No segundo caso, efeito similar é alcançado pelo fato de o poço estar localizado numa porção lateral-

Tabela 1 - Variância dos sinais de perfis originais e filtrados.

\begin{tabular}{|c|c|c|c|c|c|c|}
\hline & \multicolumn{2}{|c|}{ Variância de rhoB } & \multirow{2}{*}{$\begin{array}{c}\text { Redução } \\
\text { na } \\
\text { Variância }\end{array}$} & \multicolumn{2}{|c|}{ Variância de dT } & \multirow{2}{*}{$\begin{array}{c}\text { Redução } \\
\text { na } \\
\text { Variância }\end{array}$} \\
\hline Poço & $\begin{array}{c}\text { Sinal } \\
\text { Original }\end{array}$ & $\begin{array}{c}\text { Sinal } \\
\text { Filtrado }\end{array}$ & & $\begin{array}{c}\text { Sinal } \\
\text { Original }\end{array}$ & $\begin{array}{c}\text { Sinal } \\
\text { Filtrado }\end{array}$ & \\
\hline $\mathrm{NA01}$ & 0,14 & 0,12 & $15 \%$ & 11,40 & 8,27 & $18 \%$ \\
\hline NA02 & 0,12 & 0,09 & $42 \%$ & 12,18 & 4,53 & $63 \%$ \\
\hline NA04 & 0,13 & 0,11 & $16 \%$ & 8,27 & 5,51 & $34 \%$ \\
\hline NA07 & 0,12 & 0,09 & $19 \%$ & 10,26 & 7,09 & $41 \%$ \\
\hline NA12 & 0,16 & 0,13 & $19 \%$ & 12,04 & 10,23 & $16 \%$ \\
\hline RJS42 & 0,10 & 0,08 & $20 \%$ & 8,14 & 5,40 & $34 \%$ \\
\hline RJS 234 & 0.10 & 0,07 & $30 \%$ & 7.87 & 4,30 & $46 \%$ \\
\hline
\end{tabular}
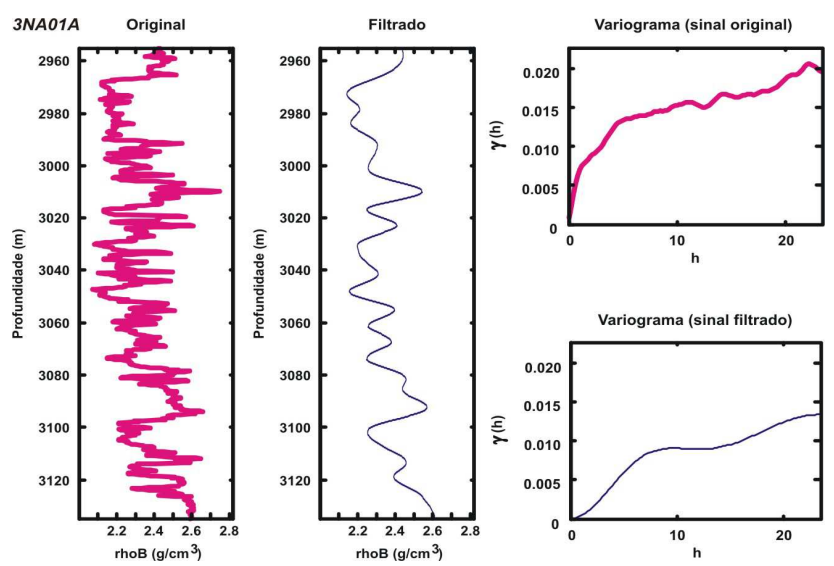

Figura 3 - Perfil original e filtrado de rhoB do poço NA01 e variograma do perfil original e filtrado.
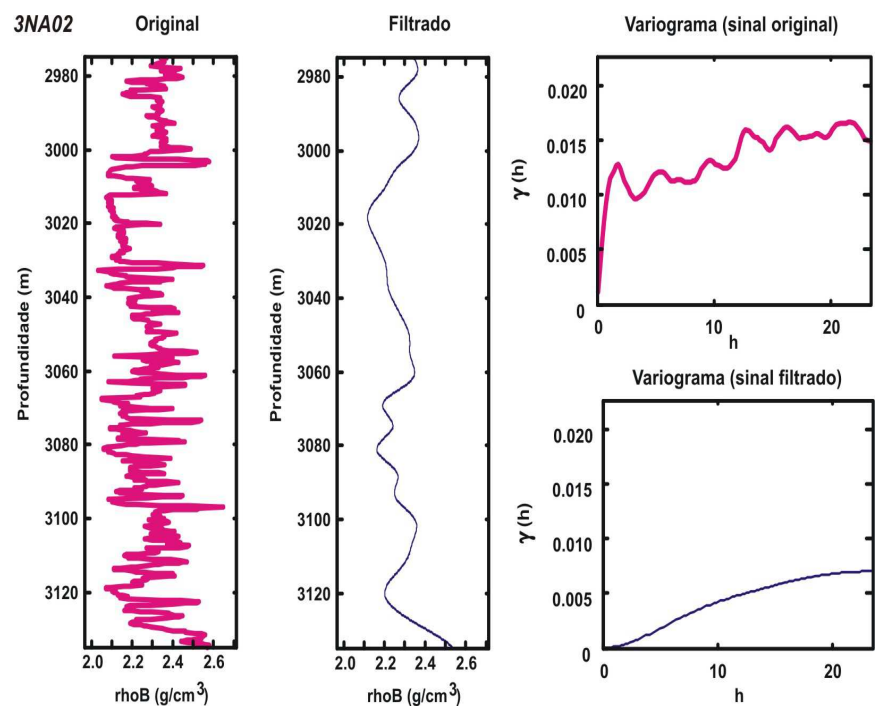

Figura 4 - Perfil original e filtrado de rhoB do poço NA02 e variograma do perfil original e filtrado.

proximal em relação aos cânions alimentadores e apresentar uma predominância de níveis argilosos finamente laminados.

Analisando a degradação do sinal sônico, observa-se que, coerentemente, nestes dois poços há uma queda acentuada na variância do perfil filtrado, fortalecendo a interpretação acima.

A redução na variância é maior, comparativamente, para dT pois o perfil sônico normalmente é mais susceptível a condições adversas de aquisição e mais ruidoso que o de densidade. A maior sensibilidade do perfil sônico é notável nos poços NA04, NA07 (Fig. 6) e RJS42 que apresentam uma queda aceitável na variância para rhoB e altos valores de redução percentual da variância para dT. É importante notar ainda que, em geral, os sinais de rhoB e dT observam forte correlação negativa, ou seja, maiores valores de densidade se refletem em menores valores de tempo de trânsito.

Além disso, a posição mais distal dos poços NA07 (Fig. 7) e RJS42, e mais lateral de NA04 implicam em intercalações mais freqüentes e de pequeno comprimento de onda que são filtradas do sinal de perfil, mesmo contendo informação geológica relevante nas freqüências acima e próximas da freqüência de corte.

Os resultados mais impressivos foram obtidos para NA01 (Fig. 


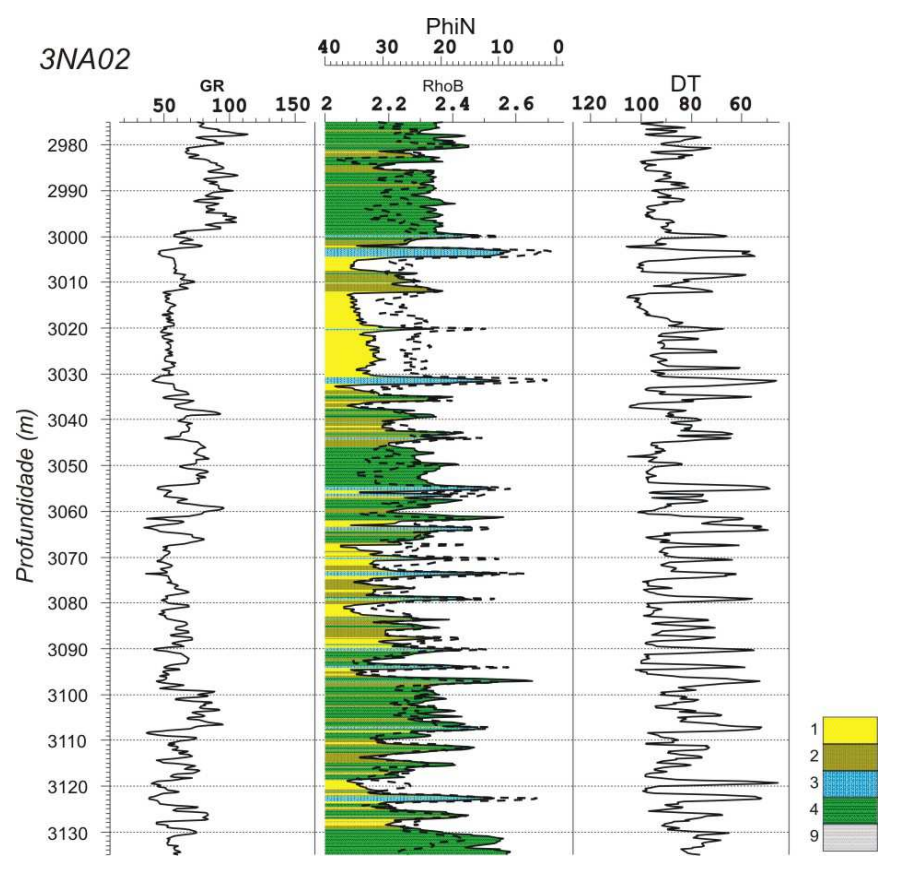

Figura 5 - Conjunto completo de perfis (raios gama, densidade, porosidade-neutrão e sônico) do poço NA02 e interpretação litológica ( 1 - arenito; 2 - arenito argiloso; 3 -arenito cimentado; 4 -folhelhos e margas; 5 - litologia não-definida). Observar as freqüentes laminações e a ocorrência de níveis cimentados.
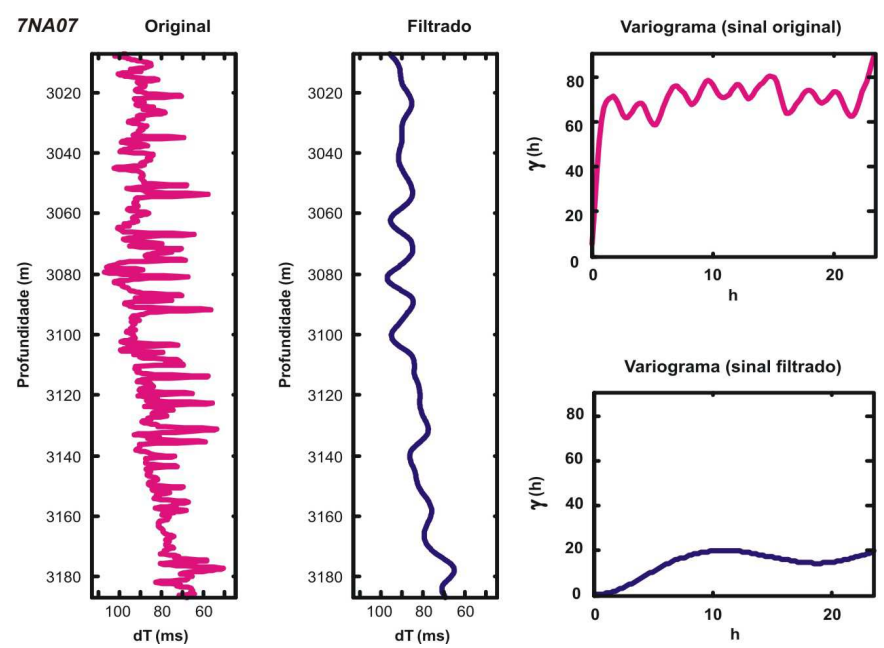

Figura 6 - Perfil original e filtrado de $\boldsymbol{d T}$ do poço NA07e variograma do perfil original e filtrado.

Figura 8-Conjunto completo de perfis do poço NA01 e interpretação litológica ( 1 - arenito; 2 - arenito argiloso; 3 arenito cimentado; 4 -folhelhos e margas; 5 - litologia nãodefinida). Observar a grande continuidade dos corpos arenosos, comparativamente com poucas intercalações de margas e de níveis cimentados.

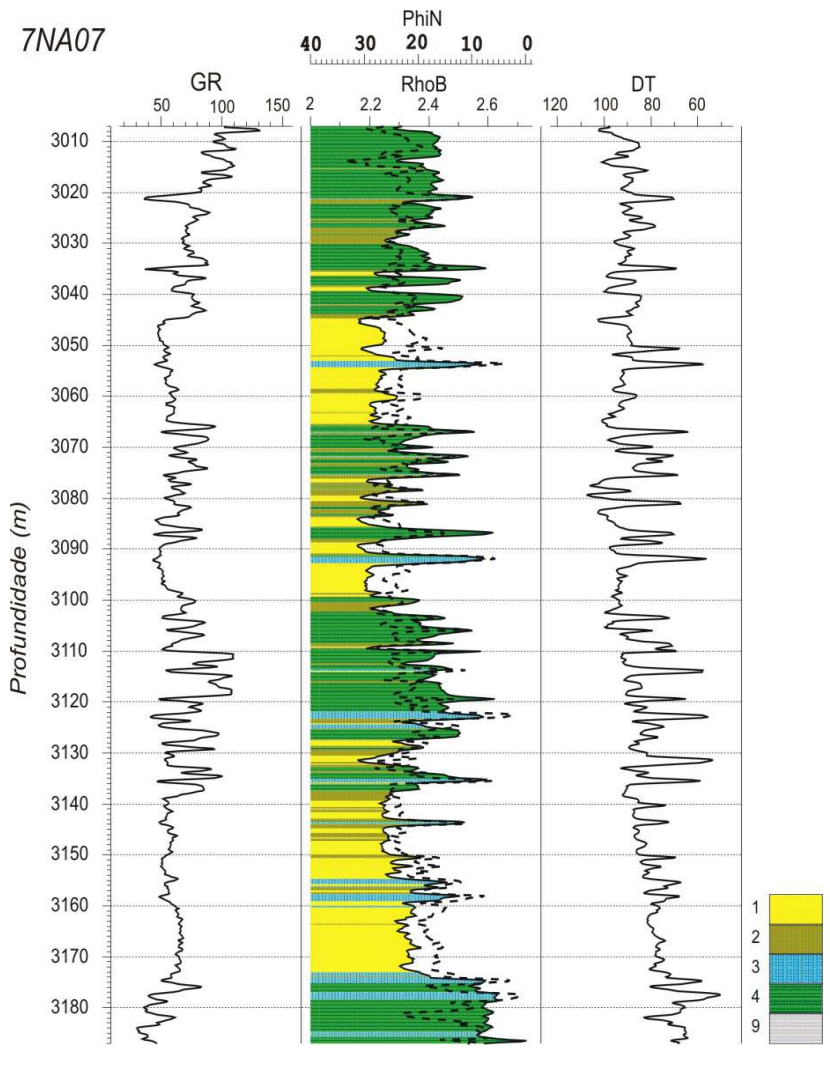

Figura 7 - Conjunto completo de perfis do poço NA07 e interpretação litológica (1 - arenito; 2 - arenito argiloso; 3 arenito cimentado; 4 -folhelhos e margas; 5 - litologia nãodefinida). Observar as laminações com pequenos comprimentos de onda, principalmente na porção central do reservatório.

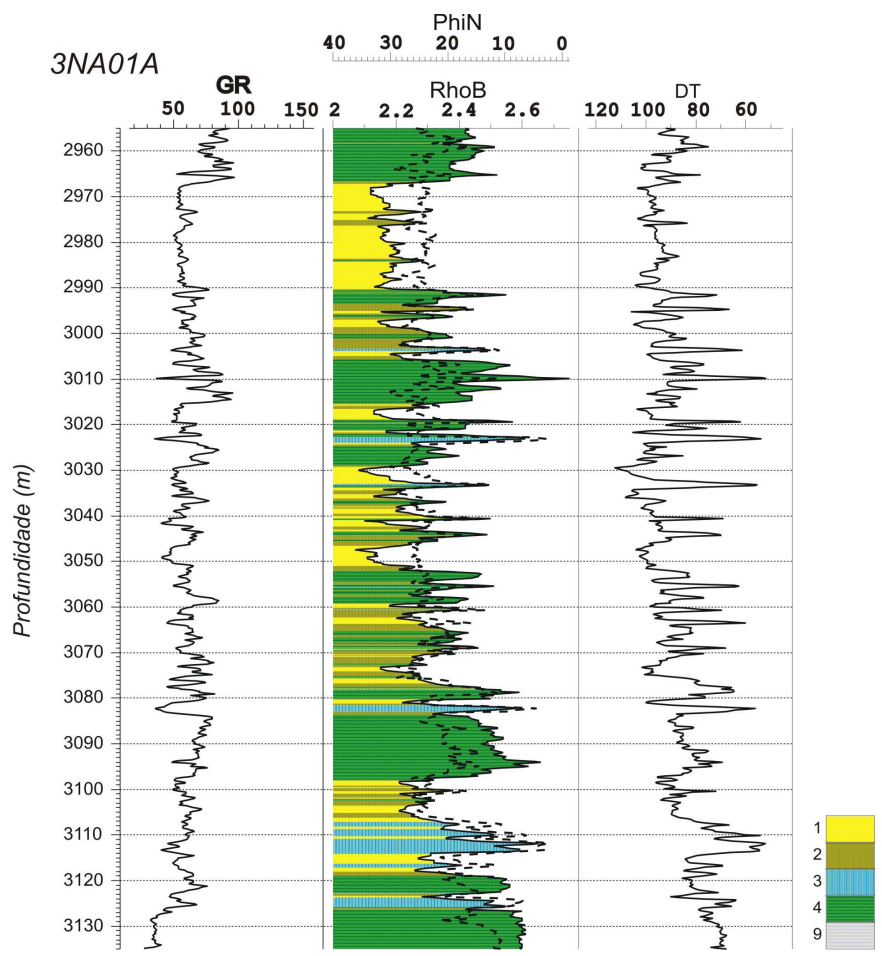


3) e NA12. Ambos ocupam uma posição central no reservatório e apresentam pacotes arenosos bem desenvolvidos, com pouca intercalação de margas e poucos intervalos cimentados (e.g., Fig. 8; Silva2 001). Como se observa na Tabela 1 , a degradação do sinal filtrado de rhoB e dT é pequena (da ordem de $15 \%$ ) e as feições principais do pacote sedimentar estão bem preservadas nesta escala. Certamente, as condições de aquisição favorecem o resultado mas a ferramenta de filtragem wavelet preserva adequadamente a qualidade do dado original, resultando numa boa representação do sinal na mudança de escala.

CONCLUSÕES ATW mostrou-se ferramenta eficaz na filtragem de sinais de perfil geofísico de poço e sua integração com informações geológicas das rochas-reservatórios fornece uma poderosa base para a mudança de escala tão importantes na modelagem de fluxos. A transferência da informação de escala submétrica dos perfis para uma escala compatível com a aquisição sísmica, cuja resolução vertical é da ordem de algumas dezenas de metros, pode ser controlada de maneira prática e eficiente ao longo das sucessivas aproximações obtidas por análise multi-resolução.

O uso do variografia no controle da filtragem e escolha de pseudo-freqüências adequadas demonstrou ser boa ferramenta no estudo desse tipo de sinal geofísico. A qualidade do produto final e sua estrutura podem ser avaliadas de maneira simples e eficaz, valorizando a informação de poço e permitindo sua utilização na geração de modelos espaciais fortemente condicionados pela informação geológica de detalhe. A transposição do sinal filtrado como núcleo elementar de modelos numéricos de reservatório, mas preservando características de pequena escala (relevantes em nível do reservatório), pode ser enriquecida com as assinaturas dos variogramas nos seus vários níveis de decomposição.

Os traços filtrados de perfil conservam parte considerável da energia do sinal original como está expresso na manutenção parcial da sua variabilidade. Os filtros wavelet efetivamente preservam a assinatura do sinal, apesar de suas características transitórias ou estacionárias. Na verdade, mesmo sinais com forte conteúdo estacionário podem ser analisados e filtrados com bons resultados utilizando-se a TW.

Agradecimentos À FAPESP- Fundação de Amparo à Pesquisa do Estado de São Paulo (Processo 99/11140-9, bolsa de pós-doutorado concedida ao primeiro autor) e à ANP- Agência Nacional de Petróleo (bolsas de Mestrado ao segundo autor e de Pesquisador-Visitante - ANP 2001.0379-0 - ao primeiro) pelo suporte financeiro a este trabalho e aos revisores da RBG pelas sugestões ao manuscrito.

\section{Referências}

Astaf'eva N.M. 1996. Wavelet Analysis: basic theory and some applications, Physics. Uspekhi, 39(11):1085-1108

Daubechies I. 1992. Ten Lectures on Wavelets. Society for Industrial and Applied Mathematics, Philadelaphia, Pennsylvania

Chu L., Schatzinger R.A., Tharn M.K. 1996. Application of wavelet analysis to upscaling of rock properties, SPE 36517, 1996

Graps, A. 1995. An Introduction to wavelets. IEEE, v. 2, n. 2

Hubbard B.B. 1998. The world according to wavelets. The story of a mathematical technique in the making. 2 ed., A. K. Peters Ltd., 286 $\mathrm{p}$.

Jansen F.E. \& Kelker M.G. 1998. Upscaling of reservoir properties using wavelets. SPE 39495

Kumar A., Farmer C.L., Jerauld G.R.Li, D. 1997. Efficient upscaling from cores to simulation models. SPE 38744 presented at the ATCE held in San Antonio, 1997.

Liu P.C. 1998. Wavelet spectrum and ocean wind waves. In: E. FourfoulaGeorgiou \& P. Kumar (eds), Wavelets in Geophysics, pp. 151-166

Mallat S. A 1989. Theory for multiresolution signal decomposition: wavelet representation. IEEE Trans. Pattern Anal. Mach. Intellig., no. 11, pp. 674-693

Meyer Y. 1989a. Wavelets and Applications. In: Proceedings of the International Conference, Marseille, France, May

Mundim E.C. 1999. Avaliação da Krigagem fatorial na filtragem de atributos sísmicos: um filtro geoestatístico aplicado à caracterização de reservatórios, Dissertação de Mestrado, Faculdade de Engenharia Mecânica, Universidade Estadual de Campinas

Oliver M.A., Bosch E., Slocum K. 2000. Wavelets and kriging for filter- ing and data reconstruction. In: W.J. Kleingeld \& D.G. Krige (eds), Geostats 2000, Cape Town

Prokoph A. \& Agtemberg, F.P. 2000. Wavelet analysis off well-logging data fro oil source rock, Egret Member, Offshore eastern Canada. AAPG Bulletin, 84(10): 1617 - 1632, 2000

Prokoph A. \& Barthelmes F. 1996. Detection of nonstationarities in geological time-series: Wavelet Transform of chaotic and ciclic sequences. Computer and Geosciences, 22(10):1097-1108

Protázio J.M.B. 2002. Análise wavelet aplicada a sinais geofisicos. Dissertação de Mestrado, Faculdade de Engenharia Mecânica e Instituto de Geociências, Universidade Estadual de Campinas

Silva A. 2001. Análise de perfis geofisicos de poço no domínio de Fourier e sua integração com a aquisição sísmica no modelamento de reservatórios. Relatório de projeto de pesquisa (processo 99/11140-9) de Pós-doc junto ao DARM - IG / UNICAMP, FAPESP

Silva A. \& Remacre A.Z. 2001. Geological constrained log filtering as a basis for scale transference, SPE 69484

Souza Jr. O.G. 1997. Stratigraphie Séquentielle et Modélisation Probabiliste des Réservoirs dún Cône Sous-marin Profond (Champ de Namorado, Brésil) - Intégration des Données Géologiques et Géophysiques. Thèse de Doctorat, Université Paris 6, Paris, 215 pp.

Torrence C. \& Compo G.P. 1978. A practical guide to wavelet analysis. Bulletin of the American Meteorological Society, 79(1):61-78

Manuscrito SR-15

Recebido em 17 de novembro de 2002 Revisão dos autores em 06 de março de 2003 Revisão aceita em 07 de abril de 2003 